\title{
Fever without source as the first manifestation of SARS-CoV-2 infection in infants less than 90 days old
}

\author{
Daniel Blázquez-Gamero ${ }^{1,2}$ (1) Cristina Epalza $^{1,2}$. José Antonio Alonso Cadenas ${ }^{3}$ - Lourdes Calleja Gero ${ }^{3}$. \\ Cristina Calvo ${ }^{2,4}$. Paula Rodríguez-Molino ${ }^{4}$. María Méndez ${ }^{5}$. Maria del Mar Santos ${ }^{2,6}$ • Victoria Fumadó ${ }^{7}$. \\ María Fernanda Guzmán ${ }^{8}$. Antoni Soriano-Arandes ${ }^{2,9}$ - Ana B. Jiménez ${ }^{10}$ - Maria Penin ${ }^{11}$ • Elvira Cobo-Vazquez ${ }^{12}$. \\ Marta Pareja ${ }^{13} \cdot$ Zulema Lobato $^{14} \cdot$ Miquel Serna ${ }^{2,15} \cdot$ Rafael Delgado $^{16} \cdot$ Cinta Moraleda $^{1,2,15} \cdot$ Alfredo Tagarro $^{2,15,17}$
}

Received: 30 October 2020 / Revised: 26 January 2021 / Accepted: 2 February 2021 / Published online: 19 February 2021

(C) The Author(s), under exclusive licence to Springer-Verlag GmbH, DE part of Springer Nature 2021

\begin{abstract}
Fever without source (FWS) in infants is a frequent cause of consultation at the emergency department, and the emergence of SARS-CoV-2 could affect the approach to those infants. The aim of this study is to define the clinical characteristics and rates of bacterial coinfections of infants $<90$ days with FWS as the first manifestation of SARS-CoV-2 infection. This is a cross-sectional study of infants under 90 days of age with FWS and positive SARS-CoV2 PCR in nasopharyngeal swab/aspirate, attended at the emergency departments of 49 Spanish hospitals (EPICO-AEP cohort) from March 1 to June 26, 2020. Three hundred and thirtythree children with COVID-19 were included in EPICO-AEP. A total of 67/336 (20\%) were infants less than 90 days old, and 27/ $67(40 \%)$ presented with FWS. Blood cultures were performed in 24/27(89\%) and were negative in all but one (4\%) who presented a Streptococcus mitis bacteremia. Urine culture was performed in 26/27(97\%) children and was negative in all, except in two (7\%) patients. Lumbar puncture was performed in 6/27(22\%) cases, with no growth of bacteria. Two children had bacterial coinfections: 1 had UTI and bacteremia, and 1 had UTI. C-reactive was protein over $20 \mathrm{mg} / \mathrm{L}$ in two children (one with bacterial coinfection), and procalcitonin was normal in all. One child was admitted to the pediatric intensive care unit because of apnea episodes. No patients died.

Conclusion: FWS was frequent in infants under 90 days of age with SARS-CoV-2 infection. Standardized markers to rule out bacterial infections remain useful in this population, and the outcome is generally good.
\end{abstract}

What is Known:

- Fever without source (FWS) in infants is a common cause of consultation at the emergency department, and young infants have a higher risk of serious bacterial infections (SBI).

- The emergence of the new coronavirus SARS-CoV-2 could affect the approach to young infants with FWS in the emergency department. Management of those children is a challenge because information about bacterial coinfection and prognosis is scarce.

What is New:

- SARS-CoV-2 infection should be ruled out in young infants ( $<90$ days of age) with FWS in areas with community transmission.

- Bacterial coinfection rarely coexists in young infants with FWS and SARS-CoV-2 infection and inflammatory markers were not increased in children without bacterial coinfection.

Keywords Coronavirus $\cdot$ SARS-CoV2 $\cdot$ COVID-19 $\cdot$ Fever without source $\cdot$ Infant $\cdot$ Serious bacterial infections

Cinta Moraleda and Alfredo Tagarro contributed equally to this work.

Communicated by Nicole Ritz

Daniel Blázquez-Gamero

danielblazquezgamero@gmail.com

Extended author information available on the last page of the article

\section{Introduction}

Fever without source (FWS) in infants is a very common cause of consultation at the emergency department (ED) [1]. Despite viral infections being the most frequent cause of FWS in children, young infants under 3 months of age have a higher risk of serious bacterial infections (SBI) than older children [1-4]. The possibility of SBI accounts for a high proportion of 
hospital admissions and antibiotic use in this age group [1, 3 , 5]. Several protocols and risk models have been developed in this population in order to individualize the management of these patients $[3,5,6]$. The emergence of the new coronavirus SARS-CoV-2 could affect the approach to infants with FWS in the ED.

The first pediatric patients with COVID-19 disease were identified in January 2020 in China, and some descriptions about disease burden in children have been published since then [7-10]. Most pediatric cases are asymptomatic, or with mild clinical manifestations [7]. Case reports and case series are widening the spectrum of COVID-19 in children, including descriptions of children with FWS [11, 12]. Management of young infants with FWS and SARS-CoV-2 infection is a challenge because information about the rate of bacterial coinfection and prognosis remains scarce $[11,12]$. The aim of this study is to describe the clinical characteristics and the rate of SBI of infants under 90 days old with FWS as the first manifestation of SARS-CoV-2 infection.

\section{Methods}

\section{Study design, setting, and population}

This is a cross-sectional study of less-than-90-days-old infants with FWS and SARS-CoV-2 infection enrolled in the Prospective Epidemiological Study of COVID-19 in Children of the Spanish Pediatric Association (EPICO-AEP), from March 1 to June 26, 2020. EPICO-AEP is a multicenter national study aiming to describe the COVID-19 in Spanish children [13]. Children younger than 18 years old, infected with SARS-CoV-2, and attended at 49 hospitals were included in this registry. For this analysis, inclusion criteria were: (i) infants under 90 days old with FWS attended at ED and (ii) positivity in reversetranscriptase polymerase chain reaction (RT-PCR) for SARS$\mathrm{CoV}-2$ in nasopharyngeal swab/aspirate.

\section{Definitions and outcome measures}

FWS was defined as axillary temperature-measured (at home and/or in ED) $\geq 38{ }^{\circ} \mathrm{C}$ in patients with a normal physical examination and no respiratory signs/symptoms or a diarrheal process. SBI was defined as isolation of relevant bacteria from blood, cerebrospinal fluid (CSF), or urine. Leukocytosis was defined as white blood cells over $15.000 / \mathrm{mm} 3$. Lymphopenia was defined as a total lymphocyte count under $3500 / \mathrm{mm} 3$ in children aged 1 week to 2 months old and 3700/mm3 in children $>60$ days of age [14]. Lymphocytosis was defined as lymphocytes over $13.100 / \mathrm{mm} 3$ in children under 2 months old and 9600/mm3 in children 2-3 months old [14].

\section{Ethics}

This study has been approved by the Ethics Committee of the coordinator hospital (Hospital Universitario 12 de Octubre, Madrid, number: 20/101) and by all the other Ethics Committees from each participating center, and informed consent was obtained from the parents or guardians.

\section{Laboratory methods}

Confirmed infection was defined as the detection of nucleic acid in RT-PCR for SARS-CoV-2. The reported sensitivity of RT-PCR for the E gene and RdRp gene assays is 5.2 and 3.8 copies per reaction at $95 \%$ detection probability, respectively [15]. Both genes needed to be amplified in RT-PCR to report a positive result. RT-PCR for SARS-CoV-2 was performed in each participating center.

\section{Statistical analysis}

Study data were standardized and prospectively collected by researchers from each center into an encoded, confidential, unique electronic database online created in REDCap (Biomed Inform, 2009) [16]. Data collected included clinical and sociodemographic variables. Continuous variables, interquartile ranges (IQR), and medians were presented in the case of non-normally distributed variables and means and standard deviations when variables were normally distributed. The denominator for each percentage was the number of subjects within the population group, without considering missing observations, unless otherwise specified. Data were analyzed using SPSS software 20.0 (IBM Corp., Armonk, NY, USA).

\section{Results}

By June 26, 336 children infected with SARS-CoV-2 had been included in the registry. A total of 67/336 (20\%) were infants less than 90 days old. Among those infants, $27 / 67$ (40\%) showed FWS as the first manifestation of SARS-CoV-2 infection. Epidemiological and clinical characteristics of these infants are summarized in Table 1 . The median age at presentation was 26 days (IQR, 16-40). Distribution of the age in days is presented in Fig. 1. Sixteen out of 27 (59\%) had household contact with a confirmed COVID-19 case (see Table 1). Five out of 27 (15\%) children had comorbidities: two were preterm infants (35 and 36 weeks of gestational age), one had congenital heart disease, one phenylketonuria, and one Swachman-Diamond Syndrome. This last was diagnosed during the admission with FWS. A total of 24/27 (89\%) were admitted to the hospital. RT-PCR for SARS-CoV-2 in nasopharyngeal swabs or aspirates detected viral nucleic acid at admission in 26/27 (93\%), and one child with negative RT-PCR had a positive RT-PCR in a second sample 
Table 1 Demographics and Clinical Characteristics at admission of 27 infants with SARS-CoV-2 infection and fever without a source

\begin{tabular}{ll}
\hline Demographic characteristics & Count and percentage $(\%)$ \\
\hline Age at presentation (days) & $26(16-40)$ \\
Male sex & $17(63)$ \\
Confirmed case in household & $16(59.3)$ \\
Both parents & $4 / 16(25)$ \\
Mother & $3 / 16(18.8)$ \\
Father & $2 / 16(12.5)$ \\
Grandparents & $4 / 16(25)$ \\
Brother/sister & $1(6.3)$ \\
Other family members & $2 / 16(12.5)$ \\
Comorbidities & $5 / 27(18.5)$ \\
Prematurity & $2 / 27(7.4)$ \\
Immunodeficiency & $1 / 27(3.7)$ \\
Congenital heart disease & $1 / 27(3.7)$ \\
Metabolic disorder & $1 / 27(3.7)$ \\
Clinical characteristics & Median (interquartile range) \\
Temperature at ED ( $\left.{ }^{\circ} \mathrm{C}\right)$ & $38.1(37.5-38.4)$ \\
Total days of fever before ED admission & $1(1-2)$ \\
Heart rate (beats per minute) & $156(144-166)$ \\
Respiratory rate per minute & $40(35-48)$ \\
Oxygen saturation with room air $(\%)$ & $98(97-99)$ \\
\hline
\end{tabular}

$E D$ emergency department

$48 \mathrm{~h}$ after admission. Seven infants had at least one RT-PCR test during the follow-up; 5/7 became negative. Two children had a negative second RT-PCR 3 and 60 days after the first positive RT-PCR. Three infants had a third negative RT-PCR after two positive RT-PCR (negative RT-PCR 10, 12, and 14 days after the first positive RT-PCR). Two children that were retested only once ( 2 days and 9 days after the first RT-PCR), the RT-PCR were still positive.

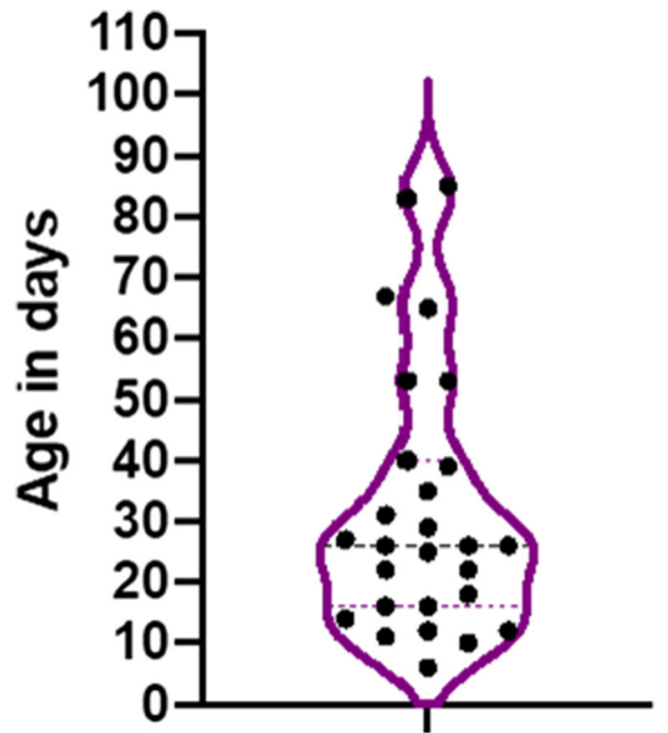

Fig. 1 Distribution of age of young infants with fever without source and SARS-CoV-2
The median temperature at admission was $38.1{ }^{\circ} \mathrm{C}(\mathrm{IQR}$, 37.5-38.4), and the median time of fever before ED admission was one day (IQR, 0-1). At admission, median transcutaneous oxygen saturation with room air was $98 \%$ (IQR, 97-99). The main laboratory results are summarized in Table 2. Median values of C-reactive protein (CRP) and procalcitonin (PCT) at admission were $1.9 \mathrm{mg} / \mathrm{L}$ (IQR, 0.6-5) and $0.12 \mathrm{ng} / \mathrm{mL}$ (IQR, $0.1-0.15)$, respectively. Only two cases presented a CRP over $20 \mathrm{mg} / \mathrm{L}$. One of them was the infant with the SwachmanDiamond Syndrome that presented bacterial coinfection described below. The second one was a 27-day-old boy with CRP of $64.8 \mathrm{mg} / \mathrm{L}$, but without bacterial infection found in any of blood, urine, or CSF cultures. No infant showed PCT over $0.5 \mathrm{ng} / \mathrm{mL}$. No infants presented with leukocytosis (median; 6010 leucocytes/mm3, IQR, 4285-8065) or lymphocytosis (median; 2900 lymphocytes/mm3, IQR, 905-2,370). Notably, 68\% (17/25) showed lymphopenia.

Chest X-rays (CXR) were done in six children because they developed respiratory symptoms during admission; of them, 3/6 (50\%) showed pulmonary infiltrates. Three of them needed oxygen supplementation with nasal cannula, but there was no correspondence between oxygen needs and the presence of infiltrates.

Blood cultures were performed in 24/27 (89\%) children and were negative in all but one, who presented a Streptococcus mitis bacteremia. The remaining three infants without blood cultures did not receive any antibiotics and showed no complications. RT-PCR for 16 respiratory viruses was performed in six children and was negative in all of them.

Urine dipstick was performed in 25/27 (93\%) infants, and $2 / 25(8 \%)$ patients showed leukocyturia. Urine culture was performed in 26/27 (97\%) children and was negative in all, except in 2 patients (7\%) patients. One child had urine culture with growth of Escherichia coli (>50.000 UFC/mL) and another, who also had a positive blood culture with growth of $\mathrm{S}$. mitis, had Enterobacter cloacae (>100,000 UFC/mL). Both samples were obtained from urethral catheterization; only the first of them showed leucocytes in urine dipstick.

Lumbar puncture was performed in 6/27 (22\%) cases, with no growth of bacteria in any case. PCRs for virus herpes simplex or enterovirus were performed in three and four patients, respectively, and all were negative. One 21-day-old girl presented pleocytosis ( 45 cells $/ \mathrm{mm}^{3}$ ). RT-PCR in CSF did not detect viral nucleic acid of SARS-CoV-2.

Therefore, 2/27 (7\%) children had bacterial coinfections detected. One child, previously mentioned, with neutropenia and Swachman-Diamond Syndrome, who presented $S$. mitis bacteremia and leukocyturia with E. cloacae in urine culture, had a CRP of $32.4 \mathrm{mg} / \mathrm{L}$. The other case was a 1-month-old infant with $E$. coli in urine culture (without leukocyturia), CRP of $4.1 \mathrm{mg} / \mathrm{L}$, and PCT of $0.2 \mathrm{ng} / \mathrm{ml}$ who was admitted to the pediatric intensive care unit (PICU) due to apnea episodes. 
Table 2 Laboratory findings at admission of 27 infants, except for alanine aminotransferase $(n=$ 16) and procalcitonin $(n=20)$

\begin{tabular}{ll}
\hline Laboratory findings & $\begin{array}{l}\text { Median (interquartile range) } \\
\text { and percentage }(\%)\end{array}$ \\
\hline White blood cell count $\left({\left.\mathrm{per} \mathrm{mm}^{3}\right)}^{3}\right.$ & $6100(4285-8065)$ \\
Total neutrophils $\left(\right.$ per $\left.\mathrm{mm}^{3}\right)$ & $1300(905-2360)$ \\
Total lymphocytes $\left(\right.$ per $\left.\mathrm{mm}^{3}\right)$ & $2900(1831-3960)$ \\
Lymphopenia & $17 / 25(68)$ \\
Platelet count (per mm $\left.{ }^{3}\right)$ & $267,000(203,000-404,000)$ \\
Hemoglobin $(\mathrm{g} / \mathrm{L})$ & $12(11.2-15)$ \\
Alanine aminotransferase $(\mathrm{U} / \mathrm{L})$ & $24(18.5-32.3)$ \\
Creatinine (mg/dL) & $0.26(0.2-0.32)$ \\
C-reactive protein $(\mathrm{mg} / \mathrm{L})$ & $1.9(0.6-5)$ \\
C-reactive protein $>20 \mathrm{mg} / \mathrm{L}$ & $2 / 27(7.4)$ \\
Procalcitonin (ng/L) & $0.12(0.1-0.15)$ \\
Procalcitonin $>0.5$ ng/L & $0 / 20(0)$ \\
Leucocytes in urine dipstick & $2 / 25(8)$ \\
Lumbar puncture performed & $6 / 27(22.2)$ \\
Pleocytosis in CSF & $1 / 4(25)$ \\
Microbiology & \\
Positive SARS-CoV-2 PCR in first nasopharyngeal swabs/aspirates & \\
Positive blood culture & $26 / 27(93)$ \\
Positive urine culture & $1 / 24(4)$ \\
Bacteria in CSF culture & $2 / 26(7.7)$ \\
Positive enterovirus PCR in CSF & $0 / 6(0)$ \\
Positive virus herpes simplex PCR in CSF & $0 / 4(0)$ \\
Positive PCR 16 respiratory virus & $0 / 3(0)$ \\
\hline & $0 / 6(0)$ \\
\hline
\end{tabular}

For microbiology results, the denominator is specified in the table. CSF cerebral spinal fluid; PCR polymerase chain reaction
Intravenous antibiotics were empirically administered in $10 / 27$ (37\%) children, being ampicillin plus gentamycin the most common combination (6/10), followed by ampicillin plus a third-generation cephalosporin $(2 / 10)$, and two children with ceftriaxone alone. In addition, one child received azithromycin as part of the SARS-CoV-2 infection treatment. No other children received any antiviral treatment.

The median duration of fever during admission was 2 days (IQR, 1-3). Six out of 27 (22\%) children required oxygen by nasal cannula because respiratory symptoms were developed during admission. None of the remainders received other respiratory support. Only the child described above with concomitant UTI by E. coli was admitted to the PICU because of the apneic episodes. He was also diagnosed with pneumonia due to the presence of radiological infiltrate, but no mechanical ventilation was needed.

Final diagnoses after discharge are FWS $(21 / 27 ; 77 \%)$, viral pneumonia $(4 / 27 ; 15 \%)$, and upper respiratory tract infection $(2 / 27 ; 7 \%)$ (Table 3$)$. No patient died or showed any sequelae at discharge.

\section{Discussion}

In this study, FWS was a common feature present in infants under 90 days of age with SARS-CoV-2 infection. First communications of COVID-19 in infants were limited to mild respiratory symptoms, but new presentations have been described as the pandemic has progressed [7, 17-21]. FWS in young infants has recently been described as part of the spectrum of COVID-19[11, 12]. As several viral infections, SARS-CoV-2 may be responsible for FWS in young infants, so SARS-CoV-2 should be ruled out in areas with community transmission $[1,3,4]$.

In this study, 2/27 (7\%) infants with FWS and SARS-CoV2 infection were associated with bacterial infections. Two UTIs were observed in two patients: one of them with apneic episodes and the other with also a $S$. mitis bacteremia. This last child was diagnosed after this episode with SwachmanDiamond Syndrome and severe neutropenia, so he had a high risk of invasive bacterial infection. Otherwise, no other SBI occurred. SBI in under 90 days old infants occurs in $5-15 \%$ of the cases in FWS $[1,3,22]$. Differentiating which children 
Table 3 Evolution and outcomes of 27 infants with fever without source

\begin{tabular}{ll}
\hline Evolution and outcomes & $\begin{array}{l}\text { Median (range) or count } \\
\text { and percentage (\%) }\end{array}$ \\
\hline Hospital admission & $24 / 27(89)$ \\
Days of admission & $4(3-6)$ \\
Total days of fever & $2(1-3)$ \\
Intravenous antibiotics & $10 / 27(37)$ \\
Supplementary $\mathrm{O}_{2}$ & $6 / 27(22.2)$ \\
Days of supplementary $\mathrm{O}_{2}$ (if required) & $3(1.75-3.75)$ \\
Pediatric intensive care unit admission & $1 / 27(3.7)$ \\
Mechanical ventilation & $0 / 27(0)$ \\
Complications & $1 / 27(3.7)$ \\
Apnea episodes & $1 / 27(3.7)$ \\
Death & $0 / 27(0)$ \\
\hline
\end{tabular}

with FWS have SBI may be challenging, mainly in young infants. Several algorithms based on analytical and clinical variables have been developed throughout the years to differentiate infants with low and high risk of having SBI [1, 3, 22]. Rapid tests to identify some of the most frequent viruses like influenza or respiratory syncytial virus are usually included in the initial approach of the FWS because SBI occurrence is significantly lower in infants with viral infections than in infants without viral infection [1, 2, 4, 23]. Accordingly, the approach to young infants with FWS in the ED during a SARS-CoV-2 pandemic or outbreak should include the detection of SARS-CoV-2 in the initial microbiological study.

On the other hand, UTI was observed in this series according to previous studies where UTI prevalence is more than $5 \%$ in infants with FWS and a confirmed viral infection. Detection of SARS-CoV-2 should not lead to automatically rule out SBI. Infants can shed respiratory viruses for several days after the disease is solved [24-27], which can induce a misclassification of children. In this study, one child tested positive for RT-PCR several days after diagnosis. It is unclear if this test means that the child was infective, or if we detected only residual nucleic acid. Some of the infants who presented positive RT-PCR at admission might have undergone the infection days before. Serological tests that would confirm this in some infants were not available.

Viral coinfections were not observed in this study. However, only six children had a test performed for other viruses different from SARS-CoV-2. Due to the exceptional situation in which the hospitals were working during the peak of the epidemic, most laboratories were overloaded, and the COVID-19 study was prioritized. The prevalence of viral coinfections in this specific population and the implications in the management at ED should be further evaluated.

In general, the evolution was good, and only one infant needed PICU admission. Three out of 6 children studied presented pneumonia in the CXR, but only the child admitted to
PICU received treatment with an agent with presumed antiviral activity against SARS-CoV-2 (azithromycin). Even in this population of young infants with FWS, SARS-CoV-2 infection presents a good outcome and a low rate of PICU admission.

Lymphopenia was a remarkable feature in this population, as observed in older children and adults [28, 29]. Lymphopenia has been suggested to be an indicator of severity in COVID-19 [28, 29]. However, due to the low rate of severe cases of this series, the association of lymphopenia with severity in infants with FWS is not evaluable.

CRP and procalcitonin were low in all children with SARS-CoV-2 infection without bacterial coinfection, except in one infant with FWS and no bacterial isolation who showed a PCR of $64 \mathrm{mg} / \mathrm{L}$ and PCT of $0.4 \mathrm{ng} / \mathrm{L}$.

CRP was almost universally low, and procalcitonin was always below $0.5 \mathrm{mcg} / \mathrm{mL}$. In this study, SARS-CoV-2 was not associated with high CRP o procalcitonin levels, unlike in other inflammatory syndromes associated with COVID-19 $[15,19]$. Therefore, acute phase reactants seem to remain useful to evaluate the risk of a potential SBI in young infants during COVID-19 outbreaks.

This study may be biased, as the SARS-CoV-2 test has not been done uniformly in young febrile infants as part of a protocol, but instead guided by the case definition determined by the national authorities in each step of the pandemic, and the best judgment of the attending physician. Therefore, some cases may have been missed. Infants with comorbidities may have been tested more often, as the high proportion of comorbidities suggest. Similarly, standardized recommendations for doing CXR at admission were not in place. This precludes any recommendation about the convenience of doing CXR in infants with FWS and SARS-CoV-2 infection. We had no information on maternal status before delivery, so congenital transmission cannot be ruled out, although it is really unlikely with current evidence. Because of the pandemic situation, 
microbiologic departments were under overwork, and threshold cycle (CT) values were not available in this study.

In conclusion, the rate of SBI among infants less than 90 days of age with SARS-CoV2 and FWS was similar to other viral infections in this age group.

Standardized markers to discard SBI seem to remain useful in this population. COVID-19 should be ruled out in infants with FWS in areas with community transmission of SARSCoV-2, but the outcome is generally good.

Abbreviations COVID-19, Coronavirus disease-2019; CRP, C-reactive protein; CXR, Chest x-ray; E. cloacae, Enterobacter cloacae; E. coli, Escherichia coli; ED, Emergency department; EPICO-AEP, Prospective Epidemiological Study of COVID-19 in Children of the Spanish Pediatric Association; FWS, Fever without source; IQR, Interquartile range; PCT, Procalcitonin; PICU, Pediatric intensive care unit; RT-PCR, Reverse-transcriptase polymerase chain reaction; S. mitis, Streptococcus mitis; SARS-CoV-2, Severe acute respiratory syndrome coronavirus 2; SBI, Serious bacterial infections

Acknowledgments We thank all the patients and families for their participation in this study, the staff members who cared for them at their personal risk in this time of the epidemic, and gathered data from all the involved Units. We would like to thank Alan Smyth for the language review. We acknowledge the rest of the EPICO-AEP Team.

Authors' contribution DB, AT, and CM conceptualized and designed the study. DB and MS performed data management and statistical analysis. $\mathrm{DB}, \mathrm{CM}$, and AT drafted the manuscript. AT, and CM conceptualized and designed the EPICO-AEP study. All co-authors enrolled participants and participated in the collection of data. All co-authors participated and were involved in the critical review of the final manuscript.

Availability of data and material No data available

Code availability Not applicable

\section{Declarations}

Ethics approval The Ethics Committee of the Hospital Universitario 12 de Octubre, Madrid, approved this study (20/101),

Consent to participate Inforrmed consent was obtained from the parents or legal guardians.

Consent to publication All authors reviewed and approved the manuscript and agreed to publish this work.

Conflict of interest Dr. Epalza reported consulting fees from Viiv and a scientific travel grant from Gilead outside the submitted work. Dr. Blázquez-Gamero reported speaking fees from MSD, Roche. No other disclosures were reported.

\section{References}

1. Arora R, Mahajan P (2013) Evaluation of child with fever without source: review of literature and update. Pediatr Clin N Am 60: 1049-1062. https://doi.org/10.1016/j.pcl.2013.06.009
2. Blaschke AJ, Korgenski EK, Wilkes J et al (2018) Rhinovirus in febrile infants and risk of bacterial infection. Pediatrics 141. https:// doi.org/10.1542/peds.2017-2384

3. Baraff LJ (2008) Management of infants and young children with fever without source. Pediatr Ann 37:673-679. https://doi.org/10. 3928/00904481-20081001-01

4. L'Huillier AG, Mardegan C, Cordey S et al (2020) Enterovirus, parechovirus, adenovirus and herpes virus type 6 viraemia in fever without source. Arch Dis Child 105:180-186. https://doi.org/10. 1136/archdischild-2019-317382

5. Woll C, Neuman MI, Aronson PL (2017) Management of the febrile young infant: update for the 21st century. Pediatr Emerg Care 33:748-753. https://doi.org/10.1097/PEC.0000000000001303

6. Gomez B, Mintegi S, Bressan S et al (2016) Validation of the "stepby-step" approach in the management of young febrile infants. Pediatrics 138. https://doi.org/10.1542/peds.2015-4381

7. Dong Y, Mo X, Hu Y et al (2020) Epidemiology of COVID-19 among children in China. Pediatrics 145. https://doi.org/10.1542/ peds.2020-0702

8. Stower H (2020) Clinical and epidemiological characteristics of children with COVID-19. Nat Med 26:465. https://doi.org/10. 1038/s41591-020-0846-Z

9. de Ceano-Vivas M, Martín-Espín I, Del Rosal T et al (2020) SARSCoV-2 infection in ambulatory and hospitalised Spanish children. Arch Dis Child 105:808-809. https://doi.org/10.1136/archdischild2020-319366

10. Lu X, Zhang L, Du H et al (2020) SARS-CoV-2 infection in children. N Engl J Med 382:1663-1665. https://doi.org/10.1056/ NEJMc2005073

11. McLaren SH, Dayan PS, Fenster DB et al (2020) Novel coronavirus infection in febrile infants aged 60 days and younger. Pediatrics. https://doi.org/10.1542/peds.2020-1550

12. Wardell H, Campbell JI, VanderPluym C, Dixit A (2020) SARSCoV-2 infection in febrile neonates. J Pediatric Infect Dis Soc. https://doi.org/10.1093/jpids/piaa084

13. Tagarro A, Epalza C, Santos M et al (2020) Screening and severity of coronavirus disease 2019 (COVID-19) in children in Madrid, Spain. JAMA Pediatr. https://doi.org/10.1001/jamapediatrics. 2020.1346

14. Comans-Bitter WM, de Groot R, van den Beemd R, et al (1997) Immunophenotyping of blood lymphocytes in childhood. Reference values for lymphocyte subpopulations. J Pediatr 130: 388-393. https://doi.org/10.1016/s0022-3476(97)70200-2

15. Corman VM, Landt O, Kaiser M et al (2020) Detection of 2019 novel coronavirus $(2019-\mathrm{nCoV})$ by real-time RT-PCR. Euro Surveill 25. https://doi.org/10.2807/1560-7917.ES.2020.25.3. 2000045

16. Harris PA, Taylor R, Thielke R et al (2009) Research electronic data capture (REDCap)-a metadata-driven methodology and workflow process for providing translational research informatics support. J Biomed Inform 42:377-381. https://doi.org/10.1016/j. jbi.2008.08.010

17. Whittaker E, Bamford A, Kenny J et al (2020) Clinical characteristics of 58 children with a pediatric inflammatory multisystem syndrome temporally associated with SARS-CoV-2. JAMA. https://doi.org/10.1001/jama.2020.10369

18. Dufort EM, Koumans EH, Chow EJ et al (2020) Multisystem inflammatory syndrome in children in New York State. N Engl J Med 383:347-358. https://doi.org/10.1056/NEJMoa2021756

19. Moraleda C, Serna-Pascual M, Soriano-Arandes A et al (2020) Multi-inflammatory syndrome in children related to SARS-CoV-2 in Spain. Clin Infect Dis. https://doi.org/10.1093/cid/ciaa1042

20. Nathan N, Prevost B, Corvol H (2020) Atypical presentation of COVID-19 in young infants. Lancet 395:1481. https://doi.org/10. 1016/S0140-6736(20)30980-6 
21. Garcia-Lara G, Linares-González L, Ródenas-Herranz T, RuizVillaverde R (2020) Chilblain-like lesions in pediatrics dermatological outpatients during the COVID-19 outbreak. Dermatol Ther e13516. https://doi.org/10.1111/dth.13516

22. Esposito S, Rinaldi VE, Argentiero A et al (2018) Approach to neonates and young infants with fever without a source who are at risk for severe bacterial infection. Mediat Inflamm 2018: 4869329. https://doi.org/10.1155/2018/4869329

23. DePorre AG, Aronson PL, McCulloh RJ (2017) Facing the ongoing challenge of the febrile young infant. Crit Care 21:68. https://doi. org/10.1186/s13054-017-1646-9

24. Principi N, Zampiero A, Gambino M et al (2015) Prospective evaluation of rhinovirus infection in healthy young children. J Clin Virol 66:83-89. https://doi.org/10.1016/j.jcv.2015.03.013

25. Loeffelholz MJ, Trujillo R, Pyles RB et al (2014) Duration of rhinovirus shedding in the upper respiratory tract in the first year of life. Pediatrics 134:1144-1150. https://doi.org/10.1542/peds.20142132

26. Kalu SU, Loeffelholz M, Beck E et al (2010) Persistence of adenovirus nucleic acids in nasopharyngeal secretions: a diagnostic conundrum. Pediatr Infect Dis J 29:746-750. https://doi.org/10. 1097/INF.0b013e3181d743c8

27. El Kholy AA, Mostafa NA, Ali AA, et al (2016) The use of multiplex PCR for the diagnosis of viral severe acute respiratory infection in children: a high rate of co-detection during the winter season. Eur J Clin Microbiol Infect Dis 35:1607-1613. https://doi.org/10. 1007/s10096-016-2698-5

28. Tan L, Wang Q, Zhang D et al (2020) Lymphopenia predicts disease severity of COVID-19: a descriptive and predictive study. Signal Transduct Target Ther 5:33. https://doi.org/10.1038/ s41392-020-0148-4

29. Huang I, Pranata R (2020) Lymphopenia in severe coronavirus disease-2019 (COVID-19): systematic review and meta-analysis. J Intensive Care 8:36. https://doi.org/10.1186/s40560-020-00453-4

Publisher's note Springer Nature remains neutral with regard to jurisdictional claims in published maps and institutional affiliations. 


\section{Affiliations}

\section{Daniel Blázquez-Gamero ${ }^{1,2}$ (D) $\cdot$ Cristina Epalza ${ }^{1,2}$ • José Antonio Alonso Cadenas ${ }^{3}$ - Lourdes Calleja Gero ${ }^{3}$. Cristina Calvo $^{2,4}$ • Paula Rodríguez-Molino ${ }^{4}$ María Méndez ${ }^{5}$ - Maria del Mar Santos ${ }^{2,6}$ • Victoria Fumadó ${ }^{7}$. María Fernanda Guzmán ${ }^{8}$ • Antoni Soriano-Arandes ${ }^{2,9}$ • Ana B. Jiménez ${ }^{10}$ • Maria Penin ${ }^{11}$ • Elvira Cobo-Vazquez ${ }^{12}$. Marta Pareja ${ }^{13} \cdot$ Zulema Lobato $^{14}$ - Miquel Serna ${ }^{2,15} \cdot$ Rafael Delgado $^{16}$ - Cinta Moraleda ${ }^{1,2,15}$ - Alfredo Tagarro ${ }^{2,15,17}$}

Cristina Epalza

crepalza@ hotmail.com

José Antonio Alonso Cadenas

jalonsocadenas@gmail.com

Lourdes Calleja Gero

lourdes.calleja@salud.madrid.org

Cristina Calvo

ccalvorey@gmail.com

Paula Rodríguez-Molino paularmolino@gmail.com

María Méndez

mariamendez24@gmail.com

Maria del Mar Santos

marimarsantos5@hotmail.com

Victoria Fumadó

Vfumado@sjdhospitalbarcelona.org

María Fernanda Guzmán

mafergm@gmail.com

Antoni Soriano-Arandes

tsorianoarandes@gmail.com

1 Pediatric Infectious Diseases Unit, Pediatric Research and Clinical Trials Unit (UPIC), Instituto de Investigación Sanitaria Hospital 12 de Octubre (imas12), Hospital Universitario 12 de Octubre, Universidad Complutense, Madrid, Spain

2 Red de Investigación Translacional en Infectología Pediátrica (RITIP), Madrid, Spain

3 Pediatrics Department, Hospital Universitario Niño Jesús, Madrid, Spain

4 Pediatric and Infectious Diseases Department, Hospital Universitario La Paz, Fundación IdiPaz, Madrid, Spain

5 Pediatric Infectious Diseases Unit, Department of Pediatrics, Hospital Universitari Germans Trias i Pujol de Badalona, Badalona, Spain

6 Hospital General Universitario Gregorio Marañón, Madrid, Spain

7 Pediatric Infectious Diseases Unit, Department of Pediatrics, Hospital Sant Joan de Déu, Barcelona, Spain

8 Grupo HM Hospitales, Madrid, Spain
Ana B. Jiménez

abjimenez@fjd.es

Maria Penin

mariapenin@yahoo.es

Elvira Cobo-Vazquez

elvicv@ hotmail.com

Marta Pareja

marta.parejaleon@gmail.com

Zulema Lobato

zulosa@gmail.com

Miquel Serna

msernapascual@outlook.com

Rafael Delgado

rafael.delgado@salud.madrid.org

Cinta Moraleda

cintamoraledaredecilla@gmail.com

Alfredo Tagarro

alfredotagarro@gmail.com
9 Pediatric Infectious Diseases and Immunodeficiencies Unit, Hospital Universitari Vall d'Hebron, Vall d'Hebron Research Institute, Barcelona Hospital Campus, Barcelona, Spain

10 Hospital Universitario Fundación Jiménez Díaz, Madrid, Spain

11 Hospital Príncipe de Asturias, Alcalá de Henares, Spain

12 Hospital Universitario Fundación de Alcorcón, Alcorcón, Spain

13 Complejo Hospitalario Universitario de Albacete, Albacete, Spain

14 Althaia, Xarxa Assistencial Universitària de Manresa, Barcelona, Spain

15 Pediatric Research and Clinical Trials Unit. Instituto de Investigación Sanitaria Hospital 12 de Octubre (imas12), Madrid, Spain, Fundación para la Investigación Biomédica del Hospital 12 de Octubre, Madrid, Spain

16 Microbiology Department, Hospital Universitario 12 de Octubre, Madrid, Spain

17 Pediatrics Department. Hospital Universitario Infanta Sofía. Pediatrics Research Group, Universidad Europea de Madrid, Madrid, Spain 